\title{
Network-based analysis reveals novel gene signatures in peripheral blood of patients with chronic obstructive pulmonary disease

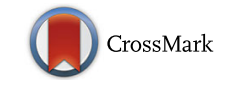

\author{
Ma'en Obeidat ${ }^{1 *}$ (D) Yunlong Nie ${ }^{1}$, Virginia Chen ${ }^{2}$, Casey P. Shannon ${ }^{2}$, Anand Kumar Andiappan $^{3}$, Bernett Lee $^{3}$, \\ Olaf Rotzschke ${ }^{3}$, Peter J. Castaldi ${ }^{4,5}$, Craig P. Hersh ${ }^{4,6}$, Nick Fishbane', Raymond T. Ng ${ }^{2}$, Bruce McManus ${ }^{1,2}$, \\ Bruce E. Miller ${ }^{7}$, Stephen Rennard ${ }^{8,9}$, Peter D. Paré ${ }^{1,10}$ and Don D. $\operatorname{Sin}^{1,10}$
}

\begin{abstract}
Background: Chronic obstructive pulmonary disease (COPD) is currently the third leading cause of death and there is a huge unmet clinical need to identify disease biomarkers in peripheral blood. Compared to gene level differential expression approaches to identify gene signatures, network analyses provide a biologically intuitive approach which leverages the co-expression patterns in the transcriptome to identify modules of co-expressed genes.

Methods: A weighted gene co-expression network analysis (WGCNA) was applied to peripheral blood transcriptome from 238 COPD subjects to discover co-expressed gene modules. We then determined the relationship between these modules and forced expiratory volume in $1 \mathrm{~s}\left(\mathrm{FEV}_{1}\right)$. In a second, independent cohort of 381 subjects, we determined the preservation of these modules and their relationship with FEV $\mathrm{F}_{1}$. For those modules that were significantly related to $\mathrm{FEV}_{1}$, we determined the biological processes as well as the blood cell-specific gene expression that were over-represented using additional external datasets.

Results: Using WGCNA, we identified 17 modules of co-expressed genes in the discovery cohort. Three of these modules were significantly correlated with $\mathrm{FEV}_{1}(\mathrm{FDR}<0.1)$. In the replication cohort, these modules were highly preserved and their $\mathrm{FEV}_{1}$ associations were reproducible $(P<0.05)$. Two of the three modules were negatively related to $\mathrm{FEV}_{1}$ and were enriched in IL8 and IL10 pathways and correlated with neutrophil-specific gene expression. The positively related module, on the other hand, was enriched in DNA transcription and translation and was strongly correlated to CD4+, CD8+ T cell-specific gene expression.
\end{abstract}

Conclusions: Network based approaches are promising tools to identify potential biomarkers for COPD.

Trial registration: The ECLIPSE study was funded by GlaxoSmithKline, under ClinicalTrials.gov identifier NCT00292552 and GSK No. SCO104960

Keywords: COPD, FEV 1 , Blood, mRNA, Gene expression, Co-expression, WGCNA, Biomarker, Transcriptome

\footnotetext{
* Correspondence: maen.obeidat@hli.ubc.ca

${ }^{1}$ The University of British Columbia Centre for Heart Lung Innovation, St

Paul's Hospital, 1081 Burrard Street, Vancouver, BC V6Z 1Y6, Canada

Full list of author information is available at the end of the article
} 


\section{Background}

Chronic obstructive pulmonary disease (COPD) is currently the third leading cause of death [1]. The disease is under genetic and environmental control with cigarette smoking being the major modifiable risk factor in the Western world [2]. COPD is characterized by chronic irreversible airflow limitation that is often accompanied by systemic inflammation [3, 4]. The two main morphologic phenotypes of COPD are small airway obstruction and emphysematous destruction and enlargement of airspaces. While the molecular mechanisms underlying the two processes may be different, COPD is diagnosed and assessed using lung function parameters; the most commonly used are the forced expiratory volume in $1 \mathrm{~s}\left(\mathrm{FEV}_{1}\right)$ and its ratio with the forced vital capacity $\left(\mathrm{FEV}_{1} / \mathrm{FVC}\right)$.

There is a huge unmet clinical need to identify clinically useful biomarkers for COPD [5]. To this end, blood biomarkers would be highly desirable since blood is very accessible. However, the main limitation of blood as a source for biomarker discovery is that its signals may not reflect the disease process in lungs, which are the predominant site of disease in COPD. Recently, a number of studies have evaluated the relationship of gene expression profiles in peripheral blood with COPD endpoints and have demonstrated some signal $[6,7]$. One major limitation of using gene expression data for biomarker discovery is the requirement for statistical stringency in determining significant expression changes. However, biologically, this traditional approach lacks intuition since genes are expressed (and function) in clusters or networks rather than as independent entities.

To address this limitation, in this study, we used weighted gene co-expression network (WGCNA) [8] to identify "modules" of co-expressed genes in peripheral blood of former smokers with COPD. We then used these modules to discover novel molecular pathways that are related to $\mathrm{FEV}_{1}$.

\section{Methods}

\section{Overall study design}

The overall study design is shown in Fig. 1. First, in the discovery cohort, using the WGCNA approach, we identified modules of strongly co-expressed genes. We then determined the association between these discovered gene expression modules and $\mathrm{FEV}_{1} \%$ predicted in the discovery cohort. Next, we determined the reproducibility of these relationships in an independent replication cohort. In both the discovery and replication cohorts, all analyses were performed with and without adjustment for cell counts in the peripheral circulation. We also determined whether the discovered co-expression patterns in the discovery cohort were preserved in the replication cohort. Finally, we used external cell-specific gene expression studies to determine whether the discovered gene expression modules

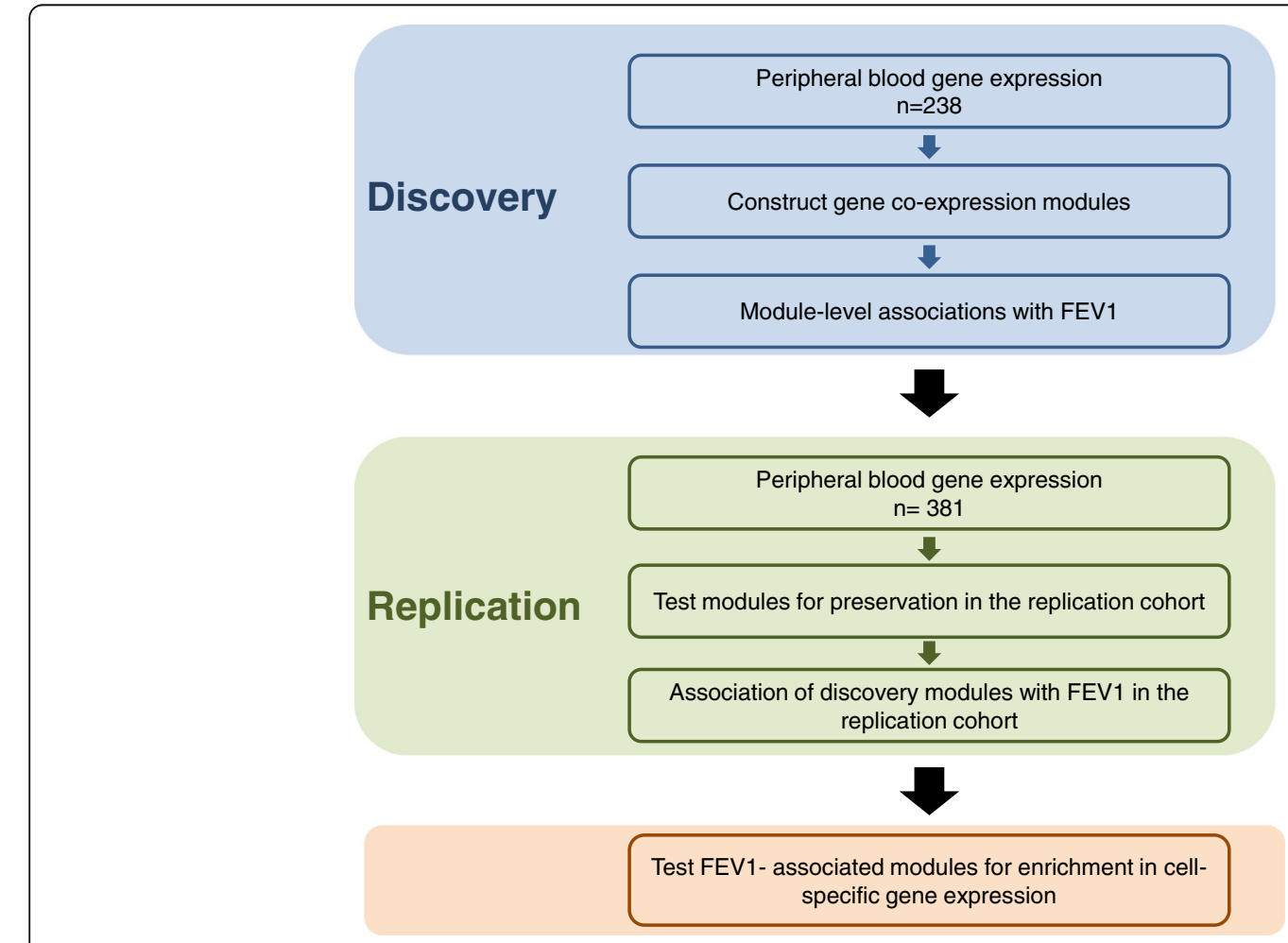

Fig. 1 Overall study design 
were enriched (i.e. over-represented) for specific cell types in the peripheral circulation (e.g. neutrophils, eosinophils, lymphocytes, monocytes, etc.).

\section{Study subjects}

The discovery and replication populations were subsets of the ECLIPSE (Evaluation of COPD Longitudinally to Identify Predictive Surrogate Endpoints) study [9]. ECLIPSE was a 3 year non-interventional, multicentre, longitudinal prospective study of COPD progression. ECLIPSE included 2164 COPD patients aged 4075 years (smoking history $\geq 10$ pack-years with a postbronchodilator $\mathrm{FEV}_{1} / \mathrm{FVC}<0.70$ and $\mathrm{FEV}_{1}<80 \%$ predicted) and 337 smokers and 245 non-smokers who were control subjects $\left(\mathrm{FEV}_{1} / \mathrm{FVC}>0.70\right.$ and $\mathrm{FEV}_{1}>90 \%$ predicted). Blood was collected in PAXgene RNA tubes and frozen at $-80{ }^{\circ} \mathrm{C}$. The gene expression sub-study of ECLIPSE was originally designed to determine gene signatures of exacerbation in peripheral blood of patients with COPD [10]. The discovery cohort consisted of 238 former smokers with COPD. The replication cohort included 381 subjects $(54.3 \%$ former and $38.6 \%$ current smokers) who were not part of the discovery set. The parent ECLIPSE study was approved by the relevant ethics review boards at each of the participating centres. Study participants provided written informed consent, and participants' information was de-identified. The ECLIPSE study was funded by GlaxoSmithKline, under ClinicalTrials.gov identifier NCT00292552 and GSK No. SCO104960. This gene expression sub-study was funded by Genome British Columbia and was approved by the Providence Health Care Research Ethics Board (REB) of the University of British Columbia (UBC) (H11-00786).

\section{Microarray data processing}

PAXgene Blood miRNA kit from PreAnalytix (Cat. \#763134) was used to extract the total RNA which was then hybridized to the Affymetrix Human Gene 1.1 ST array. Affymetrix GeneTitan MC Scanner (Affymetrix Inc.) was used to scan the array plates. The oligo Bioconductor [11] and RMA Express [12] packages were used to perform quality control on the microarray data. Background correction, normalization and summarization of the data and filtering out non-informative probe sets was undertaken using the Factor Analysis for Robust Microarray Summarization (FARMS Bioconductor package) [13]. The gene expression data are available on the NCBI Gene Expression Omnibus (GEO) under http:// www.ncbi.nlm.nih.gov/geo/query/acc.cgi?acc=GSE71220.

Weighted gene co-expression network analysis (WGCNA) The WGCNA $R$ package [8] was used to cluster groups of strongly co-expressed genes into co-expression networks. We followed the WGCNA tutorials at (http://www.genetics.ucla.edu/labs/horvath/Coexpression Network/Rpackages/WGCNA/Tutorials/index.html). A weighted gene co-expression network reconstruction algorithm was used to create co-expression networks among the unique 18,892 genes [14]. The workflow of WGCNA began by creating a matrix of Pearson correlations between genes, and transforming these into an adjacency matrix through soft thresholding by raising it to a power $\beta$. In this study $\beta=7$ was selected so that the resulting adjacency matrix approximated a scale-free topology criterion. The adjacency matrix was transformed into a topological overlap matrix (TOM) [15]. Modules were defined as groups of highly interconnected genes. To identify modules of highly co-expressed genes, we used average linkage hierarchical clustering to group genes based on the topological overlap of their connectivity, followed by a dynamic tree-cut algorithm to cluster dendrogram branches into gene modules [16]. Each of the resulting modules was assigned a color. For each gene, we calculated a Module Membership (MM) whose values ranged between 0 and 1 by correlating the gene's expression profile with the module eigengene determined by the first principal component of the gene expression profiles in that module. A gene that has a MM approaching 1 is considered to be highly connected to other genes in that module. In this study "hub" genes, which are considered to be central to the module, were defined based on the sum of ranks of their MM and gene significance for association with $\mathrm{FEV}_{1}$.

\section{Module preservation}

To test for module preservation in the replication sample, we used the $Z_{\text {summary }}$ statistics method of the WGCNA package [17]. The $Z_{\text {summary }}$ is an integrated statistics of two preservation measures: a density preservation statistic which determines whether a module genes remain highly connected in the replication network, and the connectivity based preservation statistic which determines whether the connectivity pattern between genes in the discovery network is similar to that in the replication network [17]. A permutation test is used to assess the significance of the preservation statistics and $Z_{\text {summary }}$ for the "gold" module, which is a random sample representing the entire network. Based on the thresholds determined by Langfelder et al. [17], modules with a $Z_{\text {summary }}$ score $>10$ demonstrate strong preservation.

\section{Differential gene and module expression analysis}

In the discovery dataset, linear regression was used to identify genes, whose expression was significantly related to $\mathrm{FEV}_{1} \%$ predicted, after adjustments for age, sex and pack-years of smoking. The same approach was used to 
identify modules obtained from WGCNA that were differentially expressed with regards to $\mathrm{FEV}_{1} \%$ predicted. In a sensitivity analysis, both the gene and module-level associations were adjusted for cell counts in peripheral blood. For this analysis the first three principal components from the five cell types (neutrophils \%, lymphocytes \%, monocytes \%, eosinophils \% and basophils \%) accounted for $99.7 \%$ of variation in the cell percentages and were used as covariates in the linear regression model. The Benjamini-Hochberg method was applied to correct for multiple testing [18].

\section{Replication of module FEV1 associations}

For modules identified in the discovery cohort, we computed their eigenene values in the replication cohort and then tested them for associations with $\mathrm{FEV}_{1}$ in this independent set of subjects using linear regression. Similar to the discovery cohort the analysis was adjusted for age, sex and pack-years with additional adjustment for smoking status given that the cohort consisted of former and current smokers. A parallel analysis with additional adjustment for cell count was also performed.

\section{Enrichment in cell specific gene expression}

The three modules associated with $\mathrm{FEV}_{1}$ were tested for enrichment in cell specific gene expression data from three independent studies. These include: 1) the study by Allantaz et al. [19] where they performed miRNA and mRNA expression profiling in a panel of nine human immune cell subsets (neutrophils, eosinophils, monocytes, B cells, NK cells, CD4 T cells, CD8 T cells, myeloid dendritic cells (mDCs) and plasmacytoid dendritic cells (pDCs), to identify cell-type specific expression (GSE28490 and GSE28491) in a discovery and a validation cohort, 2) the study by Naranbhai et al. [20] measured gene expression and mapped expression quantitative trait loci (eQTL) in peripheral blood CD16+ neutrophils from 101 healthy European adults (E-MTAB-3536) and 3) the study by Fairfax et al. [21] measured gene expression in $B$ cells and monocytes (E-MTAB-945).

Affymetrix arrays were normalized using RMA and Illumina arrays were normalized using quantile normalization. Non-overlapping genes across the three studies were removed. Spearman Rank correlations were performed to determine the extent of correlation between genes in significant modules and the cell specific expression values. Furthermore, permutation was performed by shuffling the expression data for 10,000 iterations and checking the number of times that the rho is greater or equal to the value obtained for each module. In addition to $P$ values, the enrichment was ranked using rho values and agreement between studies considered in the assignment of the most likely cell type.

\section{Ingenuity pathway analysis}

QIAGEN's Ingenuity Pathway Analysis (IPA', QIAGEN Redwood City, www.qiagen.com/ingenuity) was used to analyze the gene sets for enriched canonical pathways.

\section{Statistical analysis software}

All analyses were performed with $\mathrm{R}$ version 3.2.1 and Bioconductor packages [22]. Data processing was performed using Biovia Pipeline Pilot.

\section{Results}

The discovery study included 238 former smokers with COPD, while the replication cohort included 323 COPD patients and 58 controls. The demographics of study participants are shown in Table 1.

\section{Gene level associations with FEV}

At the gene level, the strongest association with $\mathrm{FEV}_{1}$ was observed for BTN2A1 (Butyrophilin subfamily 2 member A1), which was negatively correlated with $\mathrm{FEV}_{1}$ $(\mathrm{FDR}=0.094)$. It was the only gene that had an FDR $<$ 0.1 . The top 10 genes associated with $\mathrm{FEV}_{1}$ are shown in Additional file 1: Table S1.

\section{Module identifications and associations with FEV 1} Applying WGCNA to the 18,892 genes expressed in blood cells led to the identification of 17 modules of various sizes ranging from 117 in the "grey60" module to 5783 genes in the "turquoise" module. A total of 3659 genes could not be mapped to any module; these genes were grouped into the "grey" module and were not considered further in the differential expression analyses. Three modules showed strong associations with $\mathrm{FEV}_{1}$ after adjustments for age, sex and pack-years of smoking. The most significantly correlated module was the "yellow" module containing 918 genes. It had a negative relationship with $\mathrm{FEV}_{1} \quad(\mathrm{FDR}=0.004)$. The second strongest associated module with $\mathrm{FEV}_{1}(\mathrm{FDR}=0.007)$ was the "green" module which contained 553 genes and was also negatively correlated with $\mathrm{FEV}_{1}$. Finally the "brown" module which contained1569 genes was positively correlated to $\mathrm{FEV}_{1}(\mathrm{FDR}=0.03)$. The relationship between all the modules and $\mathrm{FEV}_{1}$ are shown in Table 2.

Genes in the yellow, green and brown modules showed strong enrichment for certain biological processes, suggesting that these modules have distinct biological function (Table 3). The green module, for instance, was enriched in interleukin (IL)-10, the triggering receptor expressed on myeloid cells 1 (TREM1), the Fc Receptor-mediated phagocytosis in macrophage and monocyte and the peroxisome proliferator-activated receptors (PPAR) signalling pathways. The yellow module was enriched in IL-8 signalling, the production of nitric oxide and reactive oxygen species in macrophages, and 
Table 1 Subjects demographics

\begin{tabular}{|c|c|c|c|c|c|}
\hline Variable & & Discovery & Replication & & $P$-value \\
\hline $\mathrm{N}$ & & 238 COPD & 323 COPD & 58 controls & \\
\hline Age & & $64.2 \pm 6.2$ & $63.9 \pm 6.1$ & $59.6 \pm 6.5$ & $<0.001$ \\
\hline Male & & $64.3 \%$ & $67.8 \%$ & $62.1 \%$ & 0.556 \\
\hline BMI & & $28.1 \pm 6$ & $26.5 \pm 5.8$ & $28.6 \pm 4.3$ & 0.001 \\
\hline Smoker & & & & & $<0.001$ \\
\hline & Former & $96.6 \%$ & $54.5 \%$ & $53.4 \%$ & \\
\hline & Current & $3.4 \%$ & $45.5 \%$ & $0 \%$ & \\
\hline & Never & $0 \%$ & $0 \%$ & $46.6 \%$ & \\
\hline Pack years & & $46 \pm 26.9$ & $48.2 \pm 26.4$ & $26.9 \pm 14.1$ & $<0.001$ \\
\hline FEV $1 \%$ predicted & & $49.5 \pm 16.2$ & $49.7 \pm 15.9$ & $109.7 \pm 15.8$ & $<0.001$ \\
\hline $\mathrm{FEV}_{1} / \mathrm{FVC}$ & & $0.46 \pm 0.13$ & $0.45 \pm 0.11$ & $0.79 \pm 0.06$ & $<0.001$ \\
\hline GOLD & & & & & - \\
\hline & 2 & $43.7 \%$ & $39.6 \%$ & - & \\
\hline & 3 & $44.1 \%$ & $49.2 \%$ & - & \\
\hline & 4 & $12.2 \%$ & $11.1 \%$ & - & \\
\hline Exacerbations in prior year & & $1.6 \pm 1.8$ & $0.4 \pm 0.5$ & - & $<0.001$ \\
\hline
\end{tabular}

${ }^{*} P$-value is from $\mathrm{F}$ test for continuous variables and chi-square test for categorical variables

the caveolar-mediated endocytosis and relaxin signalling pathways. The brown module was enriched in processes related to DNA transcription and translation.

\section{Mapping "hub" genes}

The identification of modules allowed mapping of hub genes, which are central to their respective modules. To

Table 2 Module associations with FEV 1 in the discovery cohort

\begin{tabular}{lrllll}
\hline Module & Estimate & SE & $p$-value & FDR & Module size \\
\hline Yellow & -59.924 & 16.106 & $2.49 \mathrm{E}-04$ & $4.49 \mathrm{E}-03$ & 918 \\
Green & -54.373 & 15.998 & $7.96 \mathrm{E}-04$ & $7.17 \mathrm{E}-03$ & 553 \\
Brown & 45.964 & 16.373 & $5.42 \mathrm{E}-03$ & $3.25 \mathrm{E}-02$ & 1569 \\
Greenyellow & -38.935 & 16.077 & $1.62 \mathrm{E}-02$ & $5.97 \mathrm{E}-02$ & 369 \\
Blue & 38.616 & 16.000 & $1.66 \mathrm{E}-02$ & $5.97 \mathrm{E}-02$ & 2399 \\
Magenta & -34.945 & 16.104 & $3.10 \mathrm{E}-02$ & $9.31 \mathrm{E}-02$ & 455 \\
Red & 30.352 & 16.007 & $5.92 \mathrm{E}-02$ & $1.52 \mathrm{E}-01$ & 510 \\
Pink & 21.163 & 16.248 & $1.94 \mathrm{E}-01$ & $4.37 \mathrm{E}-01$ & 471 \\
Turquoise & -19.410 & 16.096 & $2.29 \mathrm{E}-01$ & $4.58 \mathrm{E}-01$ & 5783 \\
Grey60 & -16.590 & 16.461 & $3.15 \mathrm{E}-01$ & $5.66 \mathrm{E}-01$ & 117 \\
Midnightblue & 9.907 & 16.225 & $5.42 \mathrm{E}-01$ & $7.14 \mathrm{E}-01$ & 152 \\
Black & 9.638 & 16.210 & $5.53 \mathrm{E}-01$ & $7.14 \mathrm{E}-01$ & 493 \\
Tan & -9.510 & 16.190 & $5.58 \mathrm{E}-01$ & $7.14 \mathrm{E}-01$ & 330 \\
Purple & -8.022 & 16.543 & $6.28 \mathrm{E}-01$ & $7.14 \mathrm{E}-01$ & 442 \\
Lightcyan & -7.684 & 16.168 & $6.35 \mathrm{E}-01$ & $7.14 \mathrm{E}-01$ & 123 \\
Salmon & -4.268 & 16.136 & $7.92 \mathrm{E}-01$ & $8.03 \mathrm{E}-01$ & 316 \\
Cyan & 4.047 & 16.209 & $8.03 \mathrm{E}-01$ & $8.03 \mathrm{E}-01$ & 233 \\
\hline
\end{tabular}

SE standard error, FDR false discovery rate identify hub genes, we used a combination of gene significance $\left(P\right.$ value) for its association with $\mathrm{FEV}_{1}$ and the gene's module membership (MM). MM is a measure of how well that gene is connected to the entire module and is reflective of a gene's centrality. Using this approach, the top hub genes for the green module were dedicator of cytokinesis 5 (DOCK5) and DENN domain containing 3 (DENND3) genes. For the yellow module, the top two hub genes were RAB3D, member RAS oncogene family (RAB3D) and GRB2 (Growth factor receptor-bound protein 2) associated binding protein 2 (GAB2). For the brown module, the top hub genes were DCAF16 and EIF2AK3. The networks of GAB2, DOCK5 and DCAF16 are shown in Fig. 1.

\section{Impact of adjustment of complete cell count (CBC) and differential to the gene and module level associations with FEV}

Because peripheral blood contains a mixture of inflammatory cells, we evaluated the impact of complete cell count $(\mathrm{CBC})$ and differential on gene expression at the gene as well as module level. The correlation of eigengenes with $\mathrm{CBC}$ in peripheral blood of the same subjects is shown in Additional file 1: Table S2 for the discovery and replication cohorts.

The yellow and green modules, which were negatively associated with $\mathrm{FEV}_{1}$ were positively correlated with neutrophils $(P<0.001)$ and negatively correlated to lymphocytes $(P<0.001)$ in peripheral blood. The brown module, which showed positive association with $\mathrm{FEV}_{1}$, was negatively correlated with neutrophils $(P<0.001)$ 
Table 3 Biological processes enrichment for the three FEV ${ }_{1}$ associated modules

\begin{tabular}{ll}
\hline Canonical Pathways & -value \\
\hline Green Module & \\
IL-10 Signaling & $4.47 \mathrm{E}-08$ \\
TREM1 Signaling & $1.14 \mathrm{E}-06$ \\
PPAR/RXR Activation & $3.96 \mathrm{E}-06$ \\
Fc Receptor-mediated Phagocytosis in & $9.75 \mathrm{E}-06$ \\
Macrophages and Monocytes & \\
PPAR Signaling & $9.75 \mathrm{E}-06$ \\
Yellow Module & \\
IL-8 Signaling & $5.90 \mathrm{E}-09$ \\
Production of Nitric Oxide and Reactive & $8.84 \mathrm{E}-07$ \\
Oxygen Species in Macrophages & \\
Caveolar-mediated Endocytosis Signaling & $2.29 \mathrm{E}-05$ \\
Role of Tissue Factor in Cancer & $2.95 \mathrm{E}-05$ \\
Relaxin Signaling & $3.26 \mathrm{E}-05$ \\
Brown Module & \\
tRNA Charging & $2.06 \mathrm{E}-10$ \\
Purine Nucleotides De Novo Biosynthesis II & $5.12 \mathrm{E}-04$ \\
Cleavage and Polyadenylation of Pre-mRNA & $8.27 \mathrm{E}-04$ \\
Nur77 Signaling in T Lymphocytes & $1.61 \mathrm{E}-03$ \\
Leucine Degradation I & $2.17 \mathrm{E}-03$ \\
\hline
\end{tabular}

and positively correlated to lymphocytes $(P<0.001)$. These relationships were replicated in the replication cohort.

To evaluate the impact of peripheral blood cell count on gene level association, the analysis was repeated by including $\mathrm{CBC}$ and differential of peripheral blood in the statistical model we had used previously. Results are shown in Additional file 1: Table S3. The smallest FDR value after cell count adjustment was 0.64 indicating that cell count adjustment had a significant effect on peripheral blood gene expression signatures for $\mathrm{FEV}_{1}$. There was a modest correlation $\left(r=0.56, P<2.2 \times 10^{-16}\right)$ of $P$ values from the cell count and the non-cell count adjusted associations.

We performed a similar analysis by adding $\mathrm{CBC}$ and differential as covariates in the module level analysis (Additional file 1: Table S4). This led to the inflation of $p$ values and a loss of statistical significance in the relationships between modules and $\mathrm{FEV}_{1}$ : the yellow and green modules, for instance, ranked third and seventh with $P$ values of 0.158 and 0.282 , respectively and an $\mathrm{FDR}=0.653$ in the $\mathrm{CBC}$ adjusted analysis.

\section{The relationship between modules and inflammatory cells in peripheral blood}

To determine which specific cell types were influencing gene expression in each of the modules, we evaluated 3 external databases that had captured cell specific gene expression in peripheral blood. The results are shown in Table 4 . The green and yellow modules, which were both negatively associated with $\mathrm{FEV}_{1}$, were enriched in neutrophils, while the brown module, which showed positive association with $\mathrm{FEV}_{1}$, was enriched in CD4+ T cells, CD8+ T cells and CD56+ NK cells.

\section{Modules' preservation and reproducibility of FEV 1 associations}

The WGCNA modules were tested for preservation in a replication cohort of 381 current and former smokers with COPD. The resulting preservation $Z_{\text {summary }}$ was $>10$, which was higher than the randomly assigned "gold" module, suggesting that all modules (except grey) were strongly preserved in the replication cohort (Fig. 2).

Table 4 Cell type enrichement for the three FEV 1 associated modules

\begin{tabular}{|c|c|c|c|c|}
\hline Reference dataset & Cell type & Module & rho* & Permutation best rho \\
\hline Allantaz et al. (Discovery) & Neutrophil & Green & 0.747 & 0.216 \\
\hline Allantaz et al. (Validation) & Neutrophil & Green & 0.715 & 0.193 \\
\hline Naranbhai et al. & Neutrophil & Green & 0.656 & 0.186 \\
\hline Allantaz et al. (Discovery) & Neutrophils & Yellow & 0.773 & 0.147 \\
\hline Allantaz et al. (Validation) & Neutrophils & Yellow & 0.729 & 0.145 \\
\hline Naranbhai et al. & Neutrophils & Yellow & 0.697 & 0.143 \\
\hline Allantaz et al. (Discovery) & CD4+ T cells & Brown & 0.618 & 0.121 \\
\hline Allantaz et al. (Discovery) & CD8+ T cells & Brown & 0.589 & 0.127 \\
\hline Allantaz et al. (Validation) & CD8+ T cells & Brown & 0.571 & 0.118 \\
\hline Allantaz et al. (Discovery) & CD56+ NK cells & Brown & 0.568 & 0.122 \\
\hline Allantaz et al. (Validation) & CD4+ T cells & Brown & 0.567 & 0.109 \\
\hline Allantaz et al. (Validation) & NK cells & Brown & 0.536 & 0.142 \\
\hline Allantaz et al. (Discovery) & CD14+ monocytes & Brown & 0.531 & 0.129 \\
\hline
\end{tabular}

*donates $P<1 \times 10^{-308}$ for all the reported Spearman's rho values. Permutation best rho: the highest rho value obtained during permutation 

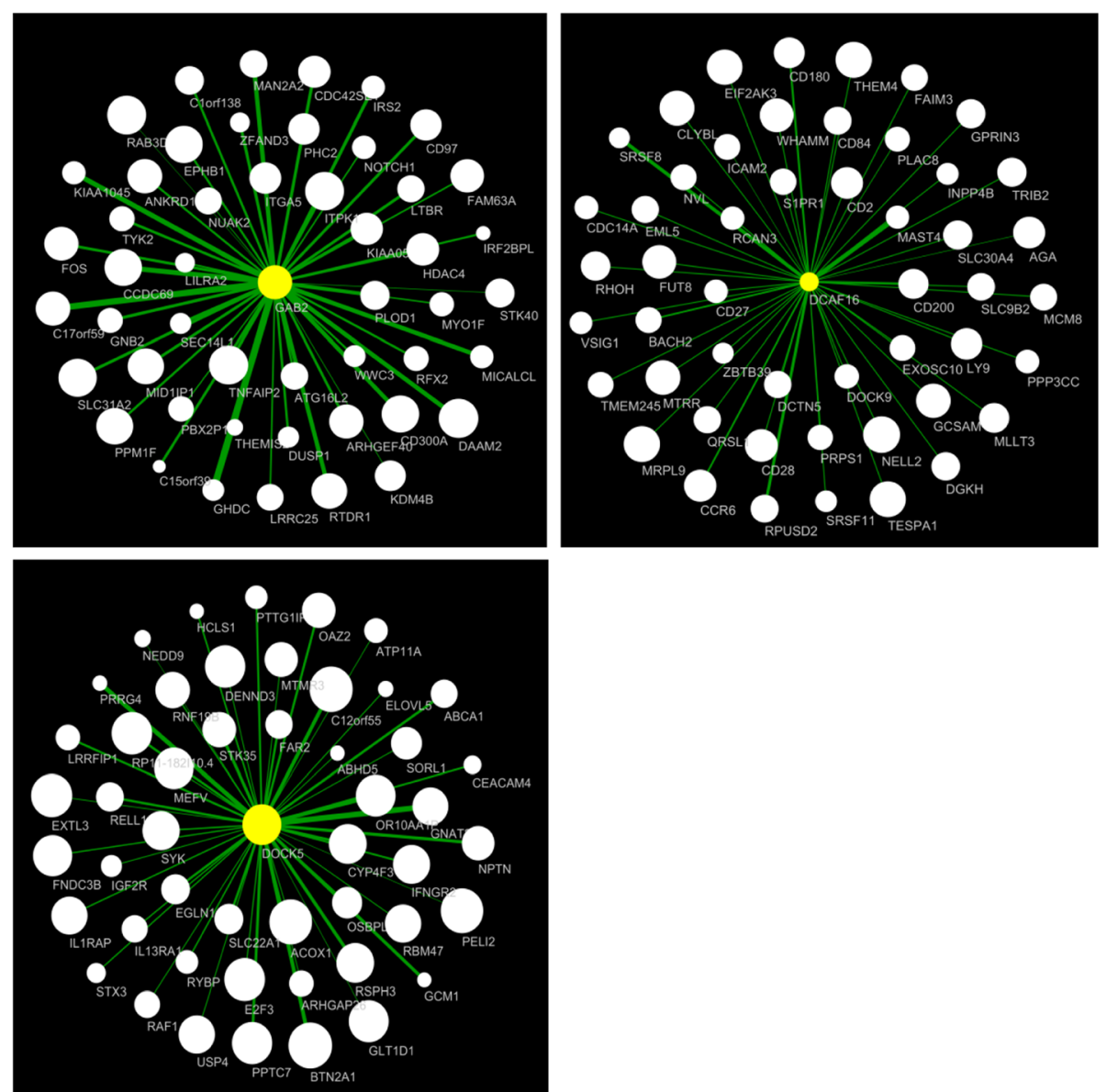

Fig. 2 Networks of GAB2, DOCK5 and DCAF16. The figure shows the networks for GAB2, DOCK5 and DCAF16 in the yellow, green and Brown modules, respectively. The genes shown are top 50 significant genes that had a FDR adjusted $P$ value $<0.05$ for association with FEV $V_{1}$. The size of the circle is proportional to the $P$ value on the $-\log 10$ scale (larger $=$ smaller $P$ value). The thickness of the edge is proportional to the topological overlap measure (TOM) identified in WGCNA

To determine whether the module associations were reproducible, eigengenes were computed in the replication cohort for modules from the discovery cohort. The new eigengenes were then tested for association with $\mathrm{FEV}_{1}$ in the replication cohort (Additional file 1: Table S5). Interestingly, the top three modules associated with $\mathrm{FEV}_{1}$ in the discovery cohort, brown, yellow and green, were also the top three modules associated with $\mathrm{FEV}_{1}$ in the replication cohort with $P=0.024, P=0.035$, and $P=0.036$ for brown, green and yellow, respectively (Figs. 3 and 4). Similar to results from the discovery cohort, adjustments for cell count in the replication cohort led to the inflation of p-values for these modules (Additional file 1: Table S6).

\section{Discussion}

COPD is an inflammatory lung disease, which has a significant systemic component that contributes to its overall morbidity and mortality. Because inflammation is thought to play a central role in the pathogenesis of COPD, there has been a tremendous surge of interest in studying circulating immune and inflammatory cells as potential biomarkers for the disease. There is a pressing need to identify genomic signatures of disease severity and activity that can guide therapeutic decisions and address the growing burden of COPD worldwide. In this study, we used modules of co-expressed genes in a highly accessible tissue, peripheral blood, to identify genomic signatures of COPD severity using $\mathrm{FEV}_{1}$ as the readout.

The main findings of the present study were that: 1) At the gene level, only one gene was associated with $\mathrm{FEV}_{1}(\mathrm{FDR}<0.1)$; 2) the 18,892 genes expressed in peripheral blood mapped to 17 modules of co-expressed genes; 3$)$ three of the modules were associated with $\left.\mathrm{FEV}_{1}, 4\right)$ in a second and larger cohort of current and former smokers with COPD and controls, all of the modules were preserved at the co-expression level, 5) the three modules in the discovery cohort that were statistically associated with $\mathrm{FEV}_{1}$ showed the strongest associations with $\mathrm{FEV}_{1}$ in the replication cohort $\left.(P<0.05), 6\right)$ the two modules, which were negatively related to $\mathrm{FEV}_{1}$, were 


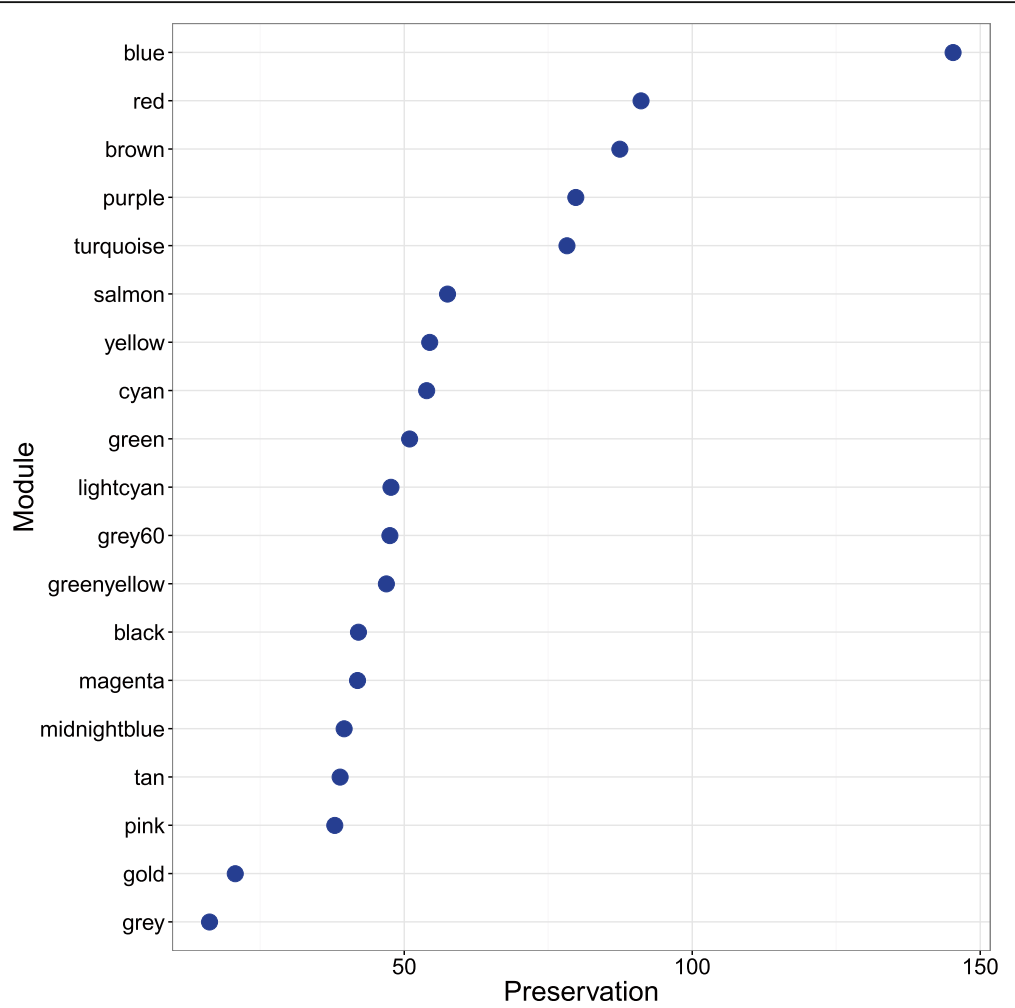

Fig. 3 Preservation $Z_{\text {summary }}$ of modules from discovery cohort in the replication cohort. The $Y$ axis shows the modules vs. their corresponding $Z_{\text {summary }}$ statistics on the $X$ axis. All modules (except the grey modules) showed a strong preservation based on the threshold prescribed in Langfelder et al. [17] of a $Z_{\text {summary }}$ score $>10$. Furthermore, the "gold" module consists of 1000 randomly selected genes that represent a sample of the whole genome, constructed for module preservation analysis. The grey module consists of genes that were not assigned to any module in the network

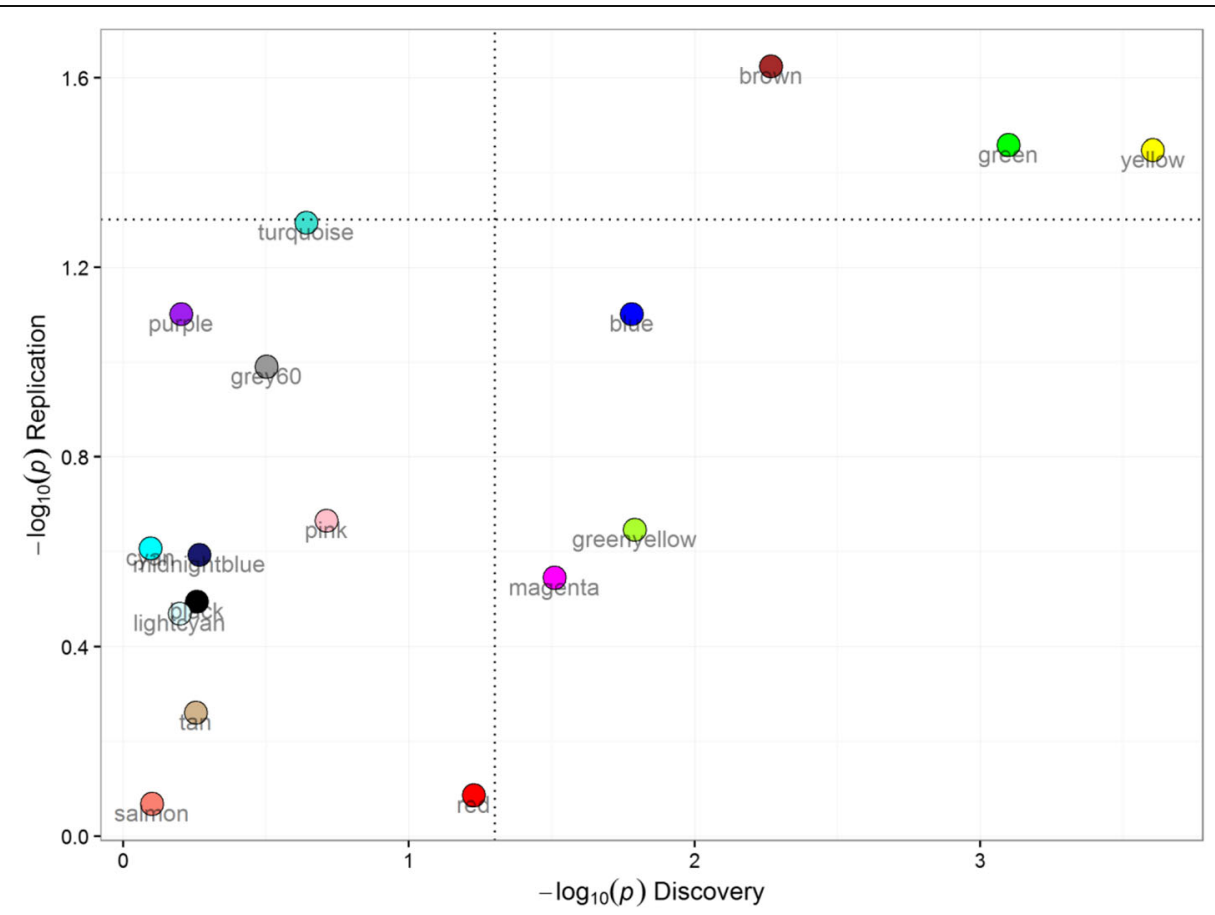

Fig. 4 Scatter plot of module associations with $\mathrm{FEV}_{1}$ in discovery and replication cohorts. The $\mathrm{Y}$ axis shows the $P$ values ( $-\log 10$ scale) for FEV 1 associations in the replication cohort while the $X$ axis shows the association $P$ values in the discovery cohort 
enriched in IL10 and IL8 pathways and were strongly correlated to neutrophil cell-specific expression, while the positively related module was enriched in DNA transcription pathways and strongly correlated to $\mathrm{T}$ cell specific expression.

Previous studies investigating differential expression in COPD have mainly tested genes and probesets individually; however, in vivo, genes are co-expressed in networks. By leveraging co-expression patterns, networks of closely co-expressed genes can be identified, often revealing novel functional pathways. The resulting network modules can then be tested for differential expression with $\mathrm{FEV}_{1}$. Another major advantage of network analyses is that this approach can significantly decrease false negatives (Type II error) by markedly reducing the number of features that are tested. In the present study the three modules reproducibly associated with $\mathrm{FEV}_{1}$ were enriched in biological pathways suggesting that co-expressed genes share biological functions within a particular module.

In each of the co-expressed networks, driver or "hub" genes can be identified, which can additionally inform the biology of these modules as they relate to $\mathrm{FEV}_{1}$. The top hub gene for the yellow module was DOCK5 which is a member of the DOCK family of guanine-nucleotide exchange factors that activate Rho-family GTPases by exchanging bound GDP for free guanosine triphosphate (GTP) [23]. DOCK5 has been shown to interact with the regulatory and catalytic subunits of protein phosphatase 2, encoded by PPP2R1A/B/C [24]. In mice, protein phosphatase $2 \mathrm{~A}$ has been shown to regulate innate immune and proteolytic responses to cigarette smoke exposure in the lung [25]. The top hub gene for the green module was $G A B 2$ which was negatively correlated to $\mathrm{FEV}_{1}$. GAB2 is a member of the growth factor receptor-bound protein 2 (GRB2) associated binding protein (GAB) gene family, which acts as an adapter molecule in signal transduction of cytokine and growth factor receptors, and $\mathrm{T}$ and B cell antigen receptors [26]. GAB2 is the principal activator of phosphatidylinositol-3 kinase in response to activation of the high affinity IgE receptor [27]. In a previous study, the expression of GAB2 in sputum was significantly increased in patients with severe emphysema compared to those who had minimal emphysema [28]. In the brown module, DDB1 and CUL4 associated factor 16 (DCAF16) and eukaryotic translation initiation factor 2 alpha kinase 3 (EIF2AK3) were the top two $\mathrm{FEV}_{1}$ hub genes. Little is known about DCAF16, and EIF2AK3 encodes a protein, which functions as an endoplasmic reticulum stress sensor [29].

Although the present study is one of the largest to date that have evaluated peripheral gene expression signature in COPD [6], at the gene level, only one gene; butyrophilin subfamily 2 member A1 (BTN2A1) was significantly associated with $\mathrm{FEV}_{1}$. Butyrophilin has been shown to regulate immune function [30]. In contrast to gene-by-gene comparison approach, the use of network based modules identified a larger number of genes within the three significant modules which were related to $\mathrm{FEV}_{1}$ highlighting the value of network approaches in identifying gene signatures. Previous work on exacerbations in COPD demonstrated similar findings [31].

It is notable that adjustments for cell count had a large impact on the relationship between gene expression signatures and $\mathrm{FEV}_{1}$. This is not surprising given that peripheral whole blood is a heterogeneous tissue composed of many different immune cell subsets. Moreover, its cellular composition varies in response to physiological or pathological processes. These processes often involve cell differentiation and/or transit of specific cell types between blood and tissues, resulting in important shifts in the cellular makeup of samples under different conditions affecting blood-derived gene expression data. Disentangling causal from reactive relationships is challenging in observational studies. Although it is common practice to statistically adjust for peripheral blood cell composition by including $\mathrm{CBC}$ and differential cell counts as covariates, regression methods do not fully take into account cell-specific gene expression and thus may obfuscate important cell-specific signatures. To explore this possibility, in the present study, in addition to the standard regression analysis, we interrogated cell-specific gene expression in three external studies that contained cell-specific gene expression data that were generated by using cell isolation methods. Using this approach, we found that the two modules which were negatively associated with $\mathrm{FEV}_{1}$, contained strong neutrophil-specific gene expression, suggesting that increased number and/or activation of peripheral neutrophils is associated with airway obstruction. The role of neutrophils in the pathogenesis of COPD is well established $[32,33]$. The module that was positively related to $\mathrm{FEV}_{1}$, on the other hand, contained gene expression signals that were $\mathrm{T}$ and $\mathrm{B}$ cell specific. Previous studies have highlighted the role of the adaptive immune response in COPD [34-37].

The current study has a number of limitations. First, gene expression signatures in peripheral blood may not reflect disease process in lungs of COPD patients. However, peripheral blood is more accessible than lung tissue and may provide information on biological processes such as immune responses that may be relevant in COPD. Second, FEV 1 may not fully capture disease activity in COPD and could reflect different pathological processes (emphysema or airway disease). Finally, the cell count adjustment had a large effect on the relationship between modules and $\mathrm{FEV}_{1}$. Given that changes in cell abundance can be causally related to changes in 
$\mathrm{FEV}_{1}$ and disease status $[38,39]$ and given the strong correlations with cell specific expression in external datasets, the regression methods used for adjustment may have been overly conservative. Most published studies to date on peripheral blood in COPD do not adjust for cell count $[6,31,40]$. Future studies are warranted that incorporate differences in cell counts and/or measurement of cell specific expression changes.

\section{Conclusions}

In conclusion, we identified gene co-expression modules in peripheral blood of patients with COPD that are highly reproducible. Three modules showed strong associations with $\mathrm{FEV}_{1}$ and were sensitive to cell count. In a larger replication cohort, the module-based co-expression patterns were preserved and associated with $\mathrm{FEV}_{1}$ in the same direction. Network based analyses represent a novel approach to discover biomarkers for COPD and warrant further attention in future studies.

\section{Additional file}

Additional file 1: Table S1. Top $10 \mathrm{FEV}_{1}$ differentially expressed genes in the discovery cohort. Table S2. Module correlations with cell counts in the discovery and replication cohorts. Table S3. Top $10 \mathrm{FEV}_{1}$ differentially expressed genes in the discovery cohort after adjusting for cell count. Table S4. Module associations with FEV 1 in the discovery cohort adjusting for cell count. Table S5. Modules association with $\mathrm{FEV}_{1}$ in the replication cohort. Table S6. Modules association with $\mathrm{FEV}_{1}$ in the replication cohort adjusting for cell counts. (DOCX $43 \mathrm{~kb}$ )

\section{Abbreviations}

COPD: Chronic obstructive pulmonary disease; ECLIPSE: Evaluation of COPD longitudinally to identify predictive surrogate endpoints; FDR: False discovery rate; $\mathrm{FEV}_{1}$ : Forced expiratory volume in $1 \mathrm{~s} ; \mathrm{FEV}_{1} \%$ pred: Percentage of predicted forced expiratory volume in $1 \mathrm{~s} ; \mathrm{FEV}_{1} / \mathrm{FVC}$ : Ratio of forced expiratory volume in $1 \mathrm{~s}$ to forced vital capacity; FVC: Forced vital capacity; MM: Module membership; PC: Principal component; WGCNA: Weighted gene co-expression network analysis

\section{Acknowledgement}

Ma'en Obeidat is a Postdoctoral Fellow of the Michael Smith Foundation for Health Research (MSFHR) and the Canadian Institute for Health Research (CIHR) Integrated and Mentored Pulmonary and Cardiovascular Training program (IMPACT). He is also a recipient of British Columbia Lung Association Research Grant.

\section{Funding}

The ECLIPSE study was funded by GlaxoSmithKline, under ClinicalTrials.gov identifier NCT00292552 and GSK No. SCO104960. This gene expression sub-study was funded by Genome British Columbia.

\section{Availability of data and materials}

The blood gene expression data are available on the NCBI Gene Expression Omnibus (GEO) under http://www.ncbi.nlm.nih.gov/geo/ query/acc.cgi?acc=GSE71220.

\section{Authors' contributions}

Conception and design of study: MO, DDS. Performed the experiments/ measurements: MO, VC, CPS, BEM, YN. Analysis and interpretation of data: $M O, V C, C P S, Y N, A K A, B L, O R$, NF. Drafting the manuscript for important intellectual content: MO, DDS, PDP, RN. Contributed to discussion: $\mathrm{CPH}$, $\mathrm{BM}$ and PC. All authors read and approved the final manuscript.

\section{Competing interests}

BEM is an employee and shareholder of GSK

SR has served as a consultant, participated in advisory boards, received honorarium for speaking or grant support from: American Board of Internal Medicine, Advantage Healthcare, Almirall, American Thoracic Society, AstraZeneca, Baxter, Boehringer Ingelheim, Chiesi, ClearView Healthcare, Cleveland Clinic, Complete Medical Group, CSL, Dailchi Sankyo, Decision Resources, Forest, Gerson Lehman, Grifols, GroupH, Guidepoint Global, Haymarket, Huron Consulting, Inthought, Johnson and Johnson, Methodist Health System - Dallas, NCI Consulting, Novartis, Pearl, Penn Technology, Pfizer, PlanningShop, PSL FirstWord, Qwessential, Takeda, Theron and WebMD. Since August 10, 2015 he has served as chief clinical scientist, new clinical development, AstraZeneca, UK.

DDS: Over the past 3 years, DDS has served as a consultant on AstraZeneca (AZ) and Novartis Advisory Boards for COPD. He has been a consultant with Amgen and Almirall. He has received research funding from AZ and Boehringer Ingelheim (BI). He has given lectures sponsored by BI and AZ.

\section{Consent for publication}

Not applicable.

\section{Ethics approval and consent to participate}

ECLIPSE study was approved by the relevant ethics review boards at each of the participating centres. Study participants provided written informed consent, and participants' information was de-identified. This gene expression sub-study was approved by the Providence Health Care Research Ethics Board (REB) of the University of British Columbia (UBC) (H1 1-00786).

\section{Publisher's Note}

Springer Nature remains neutral with regard to jurisdictional claims in published maps and institutional affiliations.

\section{Author details}

'The University of British Columbia Centre for Heart Lung Innovation, St Paul's Hospital, 1081 Burrard Street, Vancouver, BC V6Z 1Y6, Canada. ${ }^{2}$ Prevention of Organ Failure (PROOF) Centre of Excellence, Vancouver, BC, Canada. ${ }^{3}$ Singapore Immunology Network, 8A Biomedical Grove, Singapore, Singapore. ${ }^{4}$ Channing Division of Network Medicine, Brigham and Women's Hospital, Boston, USA. ${ }^{5}$ Division of General Internal Medicine and Primary Care, Brigham and Women's Hospital and Harvard Medical School, Boston, USA. 'Pulmonary and Critical Care Division, Brigham and Women's Hospital and Harvard Medical School, Boston, USA. 'GlaxoSmithKline, King of Prussia, PA, USA. ${ }^{8}$ Division of Pulmonary and Critical Care Medicine, University of Nebraska Medical Center, Omaha, NE, USA. ${ }^{9}$ Clinical Discovery Unit, Early Clinical Development, AstraZeneca, Cambridge, UK. ${ }^{10}$ Respiratory Division, Department of Medicine, University of British Columbia, Vancouver, BC, Canada.

Received: 30 October 2016 Accepted: 20 April 2017

Published online: 24 April 2017

\section{References}

1. WHO. The top 10 causes of death. Geneva: World Health Organization; 2014.

2. Vestbo J, Hurd SS, Agusti AG, Jones PW, Vogelmeier C, Anzueto A, Barnes PJ, Fabbri LM, Martinez FJ, Nishimura M, et al. Global strategy for the diagnosis, management, and prevention of chronic obstructive pulmonary disease: GOLD executive summary. Am J Respir Crit Care Med. 2013;187:347-65.

3. GOLD. Global initiative for chronic obstructive lung disease. 2011.

4. Decramer M, Janssens W. Chronic obstructive pulmonary disease and comorbidities. Lancet Respir Med. 2013;1:73-83.

5. Sin DD, Hollander Z, DeMarco ML, McManus BM, Ng RT. Biomarker development for chronic obstructive pulmonary disease. From discovery to clinical implementation. Am J Respir Crit Care Med. 2015;192:1162-70.

6. Bahr TM, Hughes GJ, Armstrong M, Reisdorph R, Coldren CD, Edwards MG, Schnell C, Kedl R, LaFlamme DJ, Reisdorph N, et al. Peripheral blood mononuclear cell gene expression in chronic obstructive pulmonary disease. Am J Respir Cell Mol Biol. 2013:49:316-23.

7. Chang Y, Glass K, Liu Y-Y, Silverman EK, Crapo JD, Tal-Singer R, Bowler R, Dy J, Cho M, Castaldi P. COPD subtypes identified by network-based clustering of blood gene expression. Genomics. 2016;107:51-8. 
8. Langfelder P, Horvath S. WGCNA: an R package for weighted correlation network analysis. BMC Bioinf. 2008:9:559

9. Vestbo J, Anderson W, Coxson HO, Crim C, Dawber F, Edwards L, Hagan G, Knobil K, Lomas DA, MacNee W, et al. Evaluation of COPD longitudinally to identify predictive surrogate end-points (ECLIPSE). Eur Respir J. 2008;31:869-73.

10. Obeidat M, Fishbane N, Nie Y, Chen V, Hollander Z, Tebbutt SJ, Bosse Y, $\mathrm{Ng}$ RT, Miller BE, McManus B, et al. The effect of statins on blood gene expression in COPD. PLoS One. 2015;10:e0140022.

11. Carvalho BS, Irizarry RA. A framework for oligonucleotide microarray preprocessing. Bioinformatics. 2010;26:2363-7.

12. Irizarry RA, Hobbs B, Collin F, Beazer-Barclay YD, Antonellis KJ, Scherf U, Speed TP. Exploration, normalization, and summaries of high density oligonucleotide array probe level data. Biostatistics. 2003;4:249-64.

13. Hochreiter S, Clevert D-A, Obermayer K. A new summarization method for affymetrix probe level data. Bioinformatics. 2006;22:943-9.

14. Zhang B, Horvath S. A general framework for weighted gene co-expression network analysis. Stat Appl Genet Mol Biol. 2005;:Article17.

15. Yip A, Horvath S. Gene network interconnectedness and the generalized topological overlap measure. BMC Bioinf. 2007;8:22.

16. Langfelder $\mathrm{P}$, Zhang $\mathrm{B}$, Horvath $\mathrm{S}$. Defining clusters from a hierarchical cluster tree: the dynamic tree cut package for R. Bioinformatics. 2008;24:719-20.

17. Langfelder P, Luo R, Oldham MC, Horvath S. Is my network module preserved and reproducible? PLoS Comput Biol. 2011:7:e1001057.

18. Benjamini $Y$, Hochberg Y. Controlling the false discovery rate: a practical and powerful approach to multiple testing. J R Stat Soc Ser B Methodol. 1995;57:289-300

19. Allantaz F, Cheng DT, Bergauer T, Ravindran P, Rossier MF, Ebeling M, Badi L Reis B, Bitter H, D'Asaro M, et al. Expression profiling of human immune cell subsets identifies miRNA-mRNA regulatory relationships correlated with cell type specific expression. PLoS One. 2012;7:e29979.

20. Naranbhai V, Fairfax BP, Makino S, Humburg P, Wong D, Ng E, Hill AVS, Knight JC. Genomic modulators of gene expression in human neutrophils. Nat Commun. 2015;6:7545.

21. Fairfax BP, Makino S, Radhakrishnan J, Plant K, Leslie S, Dilthey A, Ellis P, Langford C, Vannberg FO, Knight JC. Genetics of gene expression in primary immune cells identifies cell type-specific master regulators and roles of HLA alleles. Nat Genet. 2012;44:502-10.

22. Gentleman RC, Carey VJ, Bates DM, Bolstad B, Dettling M, Dudoit S, Ellis B, Gautier L, Ge Y, Gentry J, et al. Bioconductor: open software development for computational biology and bioinformatics. Genome Biol. 2004;5:R80.

23. Côté J-F, Vuori K. Identification of an evolutionarily conserved superfamily of DOCK180-related proteins with guanine nucleotide exchange activity. J Cell Sci. 2002;115:4901-13.

24. Glatter T, Wepf A, Aebersold R, Gstaiger M. An integrated workflow for charting the human interaction proteome: insights into the PP2A system. Mol Syst Biol. 2009:5:237.

25. Wallace AM, Hardigan A, Geraghty P, Salim S, Gaffney A, Thankachen J, Arellanos L, D'Armiento JM, Foronjy RF. Protein phosphatase $2 A$ regulates innate immune and proteolytic responses to cigarette smoke exposure in the lung. Toxicol Sci. 2012;126:589-99.

26. Hibi M, Hirano T. Gab-family adapter molecules in signal transduction of cytokine and growth factor receptors, and T and B cell antigen receptors. Leuk Lymphoma. 2000;37:299-307.

27. Gu H, Saito K, Klaman LD, Shen J, Fleming T, Wang Y, Pratt JC, Lin G, Lim B, Kinet J-P, Neel BG. Essential role for Gab2 in the allergic response. Nature. 2001;412:186-90

28. Singh D, Fox SM, Tal-Singer R, Plumb J, Bates S, Broad P, Riley JH, Celli B. Induced sputum genes associated with spirometric and radiological disease severity in COPD ex-smokers. Thorax. 2011;66:489-95.

29. Liu J, Hoppman N, O'Connell JR, Wang H, Streeten EA, McLenithan JC, Mitchell BD, Shuldiner AR. A functional haplotype in EIF2AK3, an ER stress sensor, is associated with lower bone mineral density. J Bone Miner Res. 2012;27:331-41.

30. Arnett HA, Escobar SS, Viney JL. Regulation of costimulation in the era of butyrophilins. Cytokine. 2009;46:370-5.

31. Morrow JD, Qiu W, Chhabra D, Rennard SI, Belloni P, Belousov A, Pillai SG, Hersh CP. Identifying a gene expression signature of frequent COPD exacerbations in peripheral blood using network methods. BMC Med Genomics. 2015;8:1-11.

32. Hoenderdos K, Condliffe A. The neutrophil in chronic obstructive pulmonary disease. Am J Respir Cell Mol Biol. 2013;48:531-9.
33. Gernez Y, Tirouvanziam R, Chanez P. Neutrophils in chronic inflammatory airway diseases: can we target them and how? Eur Respir J. 2010;35:467-9.

34. Faner R, Cruz T, Casserras T, Lopez-Giraldo A, Noell G, Coca I, Tal-Singer R, Miller B, Rodriguez-Roisin R, Spira A, et al. Network analysis of lung transcriptomics reveals a distinct B-cell signature in emphysema. Am J Respir Crit Care Med. 2016;193:1242-53.

35. Lloyd CM. Chair's summary: innate and adaptive immune responses in airway disease. Ann Am Thorac Soc. 2014;11:S234-5.

36. Polverino F, Cosio BG, Pons J, Laucho-Contreras M, Tejera P, Iglesias A, Rios A Jahn A, Sauleda J, Divo M, et al. B cell-activating factor. An orchestrator of lymphoid follicles in severe chronic obstructive pulmonary disease. Am J Respir Crit Care Med. 2015;192:695-705

37. Polverino F, Seys LMM, Bracke KR, Owen CA. B cells in chronic obstructive pulmonary disease: moving to center stage. Am J Physiol Lung Cell Mol Physiol. 2016;311:L687.

38. Chan-Yeung M, Abboud R, Buncio AD, Vedal S. Peripheral leucocyte count and longitudinal decline in lung function. Thorax. 1988:43:462-6.

39. Yeung MC, Buncio AD. Leukocyte count, smoking, and lung function. Am J Med. 1984;76:31-7.

40. Bhattacharya S, Tyagi S, Srisuma S, Demeo DL, Shapiro SD, Bueno R, Silverman EK, Reilly JJ, Mariani TJ. Peripheral blood gene expression profiles in COPD subjects. J Clin Bioinf. 2011:1:12.

\section{Submit your next manuscript to BioMed Central and we will help you at every step:}

- We accept pre-submission inquiries

- Our selector tool helps you to find the most relevant journal

- We provide round the clock customer support

- Convenient online submission

- Thorough peer review

- Inclusion in PubMed and all major indexing services

- Maximum visibility for your research

Submit your manuscript at www.biomedcentral.com/submit
Biomed Central 